\title{
PARAGON
}

\section{An Integrated Approach for Characterizing Aerosol Climate Impacts and Environmental Interactions}

by David J. Diner, Thomas P. Ackerman, Theodore L. Anderson, Jens Bösenberg, Amy J. Braverman, Robert J. Charlson, William D. Collins, Roger Davies, Brent N. Holben, Chris A. Hostetler, Ralph A. Kahn, John V. Martonchik, Robert t. Menzies, Mark A. Miller, John A. Ogren, Joyce E. Penner, Philip J. Rasch, Stephen E. Schwartz, John H. Seinfeld, Graeme L. Stephens, Omar Torres, Larry D. Travis, Bruce A. Wielicki, and Bin Yu

A systematic, multidisciplinary, and multiagency approach to aerosol research will accelerate scientific progress critical to understanding climate and environmental change.

A erosols are airborne suspensions of tiny particles, each typically about one-thousandth the diameter of the period at the end of this sentence. Cumulatively, they have profound effects at a variety of scales, exerting myriad influences on the earth's environment and climate and on human health. Many aerosols originate from natural sources-volcanoes, dust storms, forest and grassland fires, terrestrial and oceanic vegetation, and sea spray-whereas others arise from anthropogenic (hu- man caused) sources. The sources of anthropogenic aerosols include the burning of fossil and biofuelsthrough industrial activities, transportation systems, and urban heating-along with land cover/land-use changes, for example, biomass burning, deforestation, and desertification. Scattering and absorption of sunlight leads to a "direct" radiative forcing of climate, which, when included in model calculations, results in improved agreement with observed temperature trends over the last century (Penner et al. 2001;
AfFiliations: Diner, Braverman, Davies, Kahn, MaRTonChIK, AND MenZIES- Jet Propulsion Laboratory, California Institute of Technology, Pasadena, California; ACKERMAN-Pacific Northwest National Laboratory, Richland, Washington; ANDERSON AND CHARLSON-University of Washington, Seattle, Washington; BÖSENBERG—Max-PlankInstitut für Meteorologie, Hamburg, Germany; ColuINS AND $\mathrm{RASCH}$ - National Center for Atmospheric Research, Boulder, Colorado; HolBeN-NASA Goddard Space Flight Center, Greenbelt, Maryland; HOSTETLER AND WIELICKI-NASA Langley Research Center, Hampton, Virginia; Miller AND SCHWARTZBrookhaven National Laboratory, Upton, New York; OGRENNOAA Climate Monitoring and Diagnostics Laboratory,
Boulder, Colorado; PenNER—University of Michigan, Ann Arbor, Michigan; SEINFELD-California Institute of Technology, Pasadena, California; STEPHENS-Colorado State University, Fort Collins, Colorado; TORRES-University of Maryland, Baltimore County, Baltimore, Maryland; TRAVIS—NASA Goddard Institute for Space Studies, New York, New York; Yu—University of California, Berkeley, Berkeley, California CORRESPONDING AUTHOR: David J. Diner, JPL Mail Stop 169-237, 4800 Oak Grove Drive, Pasadena, CA 91109 E-mail: djd@jord.jpl.nasa.gov DOI:I0.II75/BAMS-85-10-I49|

In final form 28 July 2004

(C2004 American Meteorological Society 
Ramaswamy et al. 2001). Modification of cloud albedos through the seeding of smaller droplets (brightening and increase in cloud lifetimes) (Twomey 1977), or by the incorporation of absorbing aerosols (darkening) (Kaufman and Nakajima 1993), constitute "indirect" radiative forcing effects for which model uncertainties are large compared to the levels needed to assess their overall climate significance. Inhibition of regional precipitation through the modification of cloud droplet size distributions and heating profiles (Rosenfeld 2000; Ramanathan et al. 2001), adverse consequences for human respiratory health (DOE 2001; Thurston 2003, personal communication, available online at www.epa.gov/airnow/2003conference/presentations2003/GDT-HumanHealth.pdf), and degradation of visibility in parks and wilderness areas (Duce et al. 1993) are among the many ways in which aerosols affect the availability and quality of earth's water and air.

During the past decade, a number of groups have published assessments of aerosol-climate interactions and highlighted the associated uncertainties, the sources of which include

- an incomplete observational record,

- inadequate understanding of the complex physical and chemical processes involved,

- inadequate knowledge of aerosol source strengths,

- insufficient understanding of the relative importance of various mechanisms of aerosol-radiation and aerosol-cloud interactions, and

- the difficulty in representing processes that take place on submicron to subkilometer scales within climate models whose grid cells measure from tens to hundreds of kilometers in size.

A recurring recommendation of many studies ${ }^{1}$ has been the need to reduce these uncertainties through a coordinated program of local, regional, and global observations, and physical, chemical, radiation, and

\footnotetext{
${ }^{1}$ See, for example, Penner et al. (1994); Heintzenberg et al. (1996); Working Group I of the Intergovernmental Panel on Climate Change (IPCC; Penner et al. 2001; Ramaswamy et al. 2001); committees of the National Academy of Sciences (Seinfeld et at. 1996; Molina et al. 2001); Charlson (2001); the Department of Energy (DOE) Tropospheric Aerosol Program planning group (DOE 2001); the National Aerosol-Climate Interactions Program (NACIP) Scientific Steering Committee (Ramanathan et al. 2002); the World Meteorological Organization Global Atmosphere Watch Scientific Advisory Group for Aerosol (Baltensperger et al. 2003); and the Climate Change Science Program Office (Mahoney et al. 2003).
}

dynamics modeling. A major imperative is to understand the sensitivity of the climate system to increasing abundances of greenhouse gases (GHGs). Without quantifying aerosol forcing (and other critical factors, such as cloud feedbacks) to a high level of accuracy, it is not possible to evaluate the performance of climate models over the industrial period, and yet it is these same models that are relied upon for decisions regarding adaptation to a changing climate and/or mitigation of the consequences.

The complexity of the aerosol-climate problem implies that no single type of observation or model is sufficient to characterize the current system or to provide the means to predict aerosol impacts in the future with high confidence. Consequently, information must be drawn from multiple observational and theoretical techniques, platforms, and vantage points, and strategies that explicitly plan for the integration and interpretation of the various components need to be designed, more specifically, as follows:

- Remote sensing can measure the three-dimensional distribution, radiative impact, and spatial context of entire air masses, and satellites provide both regional and global views. However, determining the anthropogenic component of aerosol radiative forcing requires knowledge of sizeresolved composition that exceeds current satellite capabilities.

- Passive remote sensing methods either from a satellite (in backscatter) or from the surface (in forward scatter) are individually subject to indeterminacies that make it difficult to separate the effects of variables such as particle shape, composition, internal heterogeneity, absorptivity, abundance, size distribution, and vertical distribution. Combinations of techniques are required.

- Active techniques, such as lidar, are excellent at constraining certain properties (notably vertical distribution), but typically provide point (from the ground) or single-track (from orbit) profiling measurements only. Lidars need to be used in conjunction with passive measurements and models.

- Chemical composition must be known in order to understand the processes relating aerosol optical properties and concentrations (and ultimately, radiative forcing) to emissions. However, current spaceborne techniques seem capable of inferring only rough proxies, such as the complex refractive index. In situ airborne sensors provide detailed information about particle microphysics and chemical composition that are unachievable through other means, but their coverage is limited. 
- Atmospheric models, such as general circulation models (GCMs) and chemical transport models (CTMs) provide global diagnostics and forecasts that can calculate aerosol compositional and, increasingly, microphysical properties that are difficult to determine observationally. While GCMs incorporate some of the important aerosol physics, they do not provide the real-time meteorological consistency that is inherent in CTMs that use assimilated or forecasted meteorology; the latter, on the other hand, may ignore feedbacks between atmospheric chemistry and meteorology.

- Integration of measurements across nonuniform spatial and temporal sampling, scales, and coverages is required, using synthesis methods to achieve consistency among models and observations. Significant effort needs to be expended in data and model intercomparison, and validation to gauge the reliability of the results, and process models are required to capture the underlying physics. Practical means of interpreting the resulting multidimensional, multivariate, and, in some cases, massive informational databases are needed.

The principal points to be emphasized are that 1) many relevant and diverse data sources exist, yet the aerosol community does not currently have the means to combine them into an integrated dataset for maximum scientific benefit, and 2) a long-term solution must have the ability to bridge observational gaps and adapt to changes in measurement approaches, while maintaining consistently well-understood accuracies. Heintzenberg et al. (1996) state the issues well:

From the diverse nature of the activities ... it is evident that it is necessary to consider the problem of how to integrate them, e.g. so that the data from one activity are relevant to the others. Indeed, almost all of the individual activities are currently proceeding in a fashion that makes each one totally independent of the others. It is hardly surprising that most of the in situ data currently being acquired are of little use in climate models because there has been no intentional development of an aerosol/climate data set. It will be necessary to develop overtly an integrating activity that continuously couples the individual approaches and assumes an optimised output of results.

The assessment by Demerjian (2000) is also germane. Although his comments relate specifically to air quality information, they also speak to a more general concern:
What is arguable is whether or not the greatest advantage is being drawn from the data collected and are the data collected of sufficient quality to meet the diverse needs of the interested user communities ... A major impediment historically has been limitations associated with data distribution and analysis ... The distribution of quality assured data . . . over the Internet for all interest[ed] parties to access and use should be in place now, but it is not. The benefits of immediate open access to air quality network data are enormous. It . . . stimulates the various user communities into exercising the data and ... stimulates the development of diagnostic tools and analysis approaches ... Considering the investments ... the costs associated with implementing this needed infrastructure are trivial.

The aerosol community needs a vehicle to facilitate the transformation of information into knowledge, and ideas into action. A systematic, coordinated approach to aerosol and climate research has already been suggested (e.g., P. J. Adams 2003, personal communication, available online at www.ccsm.ucar.edu/ news/ws.chemclim.2003/adams.ppt; Charlson 2001; Trenberth et al. 2002). In response to this need, we outline an integrated program that merges multiple observational techniques, modeling capabilities, geospatial statistics research, and high-performance information technologies into a systematic framework. We have named this concept the Progressive Aerosol Retrieval and Assimilation Global Observing Network (PARAGON). Our goal is to capitalize on the intellectual heritage represented by the studies enumerated above, and PARAGON is conceived as a pathway for implementing their recommendations. Although we focus upon aerosol-climate interactions, many aspects of the approach can be applied to other problems, for example, the impact of aerosols on air quality, or the study of cloud-climate forcing and feedbacks.

OVERVIEW. This paper provides an overview of the PARAGON strategy. Supporting details are contained in a set of four companion papers, the contents of which are summarized below. Seinfeld et al. (2004) discuss the scientific motivations underlying PARAGON. The roles, strengths, and limitations of the relevant data sources are presented in Kahn et al. (2004). One key focus of PARAGON, integrating and interpreting observations and models, is addressed by Ackerman et al. (2004), while the other, establishing an accurate, consistent, and cohesive long-term record, is discussed by Diner et al. (2004). For the sake of 
brevity, references are included only in the companion papers. The appendix, "Definitions of frequently used terms," provides a guide to key expressions used throughout.

Motivations for PARAGON. PARAGON is motivated by the need to understand the sensitivity of the climate system to changes in atmospheric constituents, to reduce uncertainties in climate models, and to interpret and integrate a diverse collection of data pertaining to atmospheric aerosols. As noted by many researchers, the uncertainty in anthropogenic aerosol forcing from each of the direct and indirect effects is of the same magnitude as the forcing itself and is the dominant source of uncertainty in estimates of anthropogenic climate forcing (e.g., Penner et al. 2001; Ramaswamy et al. 2001). Reducing model uncertainties is the only way to obtain reliable inputs to decisions regarding climate adaptation and mitigation. Seinfeld et al. (2004) discuss the measurements needed, and identify four challenges that must be addressed to accomplish this objective. These challenges are summarized below.

DATA DIVERSITY. Integrating multivariate and multidimensional information from diverse sensors and models is necessary to adequately characterize aerosols. These data have nonuniform spatial and temporal sampling and coverage, and originate from many sources. Neither in situ observations, remote sensing, nor three-dimensional numerical modeling captures all essential information at all relevant scales. A comprehensive description of the global aerosol system requires cohesion of validated data from a variety of sources, and a strategy is needed for identifying, prioritizing, and obtaining measurements in undersampled regions.

DATA QUALITY LIMITATIONS. The current level of accuracy and consistency among aerosol observations and models is insufficient to meet the needs of climate research, and there are critical observational gaps. No unified plan exists to generate a cohesive, long-term aerosol record with consistent quality and accuracy. Various future climate scenarios differ by $1-2 \mathrm{~W} \mathrm{~m}^{-2}$ in the global average in their predictions of aerosol forcing. This is a fraction of a percent of the insolation received by the earth. Obtaining radiometric measurements having adequate accuracies represents a significant observational challenge, even when great care is paid to instrumental calibration. The finite duration of satellite missions, with different sensors employing different techniques, makes it difficult to separate tech- nology, calibration, and algorithm evolution from climate trends. With some exceptions (e.g., surface solar radiometers and in situ particle samplers), there are few long-term standards with which to evaluate product accuracies.

Aerosol mesoscale VARIABILITY. The inhomogeneity of aerosol sources and sinks, coupled with their relatively short atmospheric residence time, implies that the estimation of global climate forcing by aerosols must involve integrating highly variable quantities over space and time. A practical framework for merging data acquired over a wide span of spatial and temporal scales needs to be established. Spatial and temporal variability are primary causes of the large uncertainty in calculations of aerosol climate forcing. The mesoscale variability of tropospheric aerosols occurs on scales finer than generally resolved by global CTMs and by measurements with long sample times. For example, chemical samples accumulated on a 24 -h or longer basis do not always represent instantaneous aerosol properties measured from satellites. Currently, the paucity of in situ data aloft prevents the exploration of key processes at subgrid scales.

CLOUd UBIQUITY AND HETEROGENEITY. Because clouds complicate the aerosol retrieval process, and are major climate-forcing agents, conclusions regarding aerosol-induced climate and environmental changes require simultaneous cloud data, along with a means of isolating aerosol-cloud interactions from other meteorological variability. Effective cloud screening is essential for determining direct aerosol forcing. Moreover, microphysical characterizations appear inadequate to explain the angular distribution of top-of-atmosphere radiances for most cloud fields, and three-dimensional radiative transfer theory is required to account for the effects of cloud morphology, intercloud illumination, and shadowing. This is significant because remotely sensed radiances are used to infer cloud properties, such as optical depth. Isolating indirect forcing effects can be complicated by scattered light from nearby clouds, the effects of subpixel clouds, and meteorological correlations between cloudiness and pollution. Thus, even with improvements in how satellite data are modeled, satellite observations alone are insufficient to quantify aerosol indirect effects.

Data sources, roles, and attributes. Data can be obtained either observationally or from models, and fall into two broad classes: routinely generated (monitoring) and episodically generated (localized in space and/or time). The first type can be used for continuous evalu- 
ation of an assimilation model, and the second can provide essential diagnostics of model performance and indicators of model deficiencies. Because physical and chemical processes occurring on microphysical scales influence aerosol regional and global climatic and environmental impacts, linkages between data acquired on vastly different spatial and temporal scales must be made in order for transport and evolution models to capture aerosol transformation processes with high fidelity. Consistency of observations over time is also essential for the detection of long-term change. Kahn et al. (2004) review the sources of data that need to be brought together to support the goals of the PARAGON initiative. Understanding the relative strengths and limitations of such data sources is a prerequisite to establishing an effectively integrated program.

Integrating and interpreting observations and models. Data aggregation and synthesis are required to organize satellite data together with concurrently acquired in situ and surface-based remote sensing data. The approach must be adaptable to a changing measurement complement. As new data capture increasingly detailed descriptions of aerosol properties and distributions, an evolving level of sophistication will make it possible to tackle problems of increased difficulty, such as indirect forcing. Specific recommendations are presented below, with further details provided in Ackerman et al. (2004).

DATA INTEROPERABILITY AND COMPUTATIONAL INFRASTRUCTURE. A worldwide "aerosol virtual observatory" needs to be assembled to promote the widespread exchange and use of data, and take advantage of high-performance computing to enhance processing power for global modeling. The first step in making data widely accessible is to assemble an organizational infrastructure. Modern information technology approaches will benefit PARAGON through the use of "grid" and other high-performance computing initiatives to establish a distributed aerosol science information system, or "virtual observatory." Precedents in other disciplines are funded by the National Aeronautics and Space Administration (NASA), National Science Foundation (NSF), and DOE, examples of which are NASA's Information Power Grid; NSF's National Virtual Observatory, international Virtual Data Grid Laboratory, and Grid Physics Network; and DOE's Particle Physics Data Grid. The aerosol community can capitalize on this experience to apply software services, known as "middleware," to facilitate data access and collaborations; use advanced network technologies with terabits per second throughput to facilitate dataintensive computing; apply enhanced computational power to global modeling; and enable real-time visualization of complex scientific simulation results in multiple remote locations. Massively parallel or other high-performance computing approaches could be explored as a potential means of improving the spatial and temporal resolution of global models as a complement to subgrid-scale parameterizations.

Data INTEGRATION. Methodologies need to be developed for integrating observational and model data having diverse spatial and temporal sampling, resolution, and coverage. Given the diverse nature of aerosol measurements, data synthesis (combining measurements representing averages over different extents in space and time) provides the key to generating spatiotemporal aggregates spanning a multiplicity of scales. Geospatial statistics methods, such as Bayesian hierarchical modeling, provide a rigorous, data-driven approach to this problem. Another approach is assimilation, which incorporates models of aerosol physics. The latter are required in order to use the aggregated data to make forecasts based on observations. An assimilation model must have a complete specification of the aerosol sources by type and location, physical equations to describe modification due to aging and humidity changes, and removal terms due to wet and dry deposition. Data assimilation can use an assembled aerosol dataset directly, or use the integrated data provided by the geospatial statistics framework.

MUltisensor ALGORITHM DeVelopment. Algorithm development and validation need to be invested in to support joint retrievals using data from existing and future satellite, surface-based, and in situ sensors. Two examples of the potential benefits of combining data from multiple sensors as part of a joint retrieval process include 1) the simultaneous use of satellite- and surface-based multiangle measurements of scattered radiance and 2) the combination of passive and active sensor data. The first enables improved estimates of aerosol single scattering albedo, and data required to explore this approach are currently available. The second makes use of future satellite lidar/radiometer combinations to identify thin cirrus and measure aerosol variability near land-ocean boundaries, where passive techniques typically transition between different retrieval algorithms. Equally important, passive measurements of optical depth enhance lidar data because the retrieval of aerosol extinction from a simple backscatter lidar is underconstrained. A goal is to establish the accuracy with which combined 
active-passive methods can quantify and separate fine- and coarse-mode aerosol extinction, with the higher-level motivation being to assess the degree to which this separation helps constrain anthropogenic fraction.

DATA SUMMARIZATION AND MINING. Data summarization and mining techniques need to be explored to identify and interpret patterns of aerosol-induced change. Describing the observed evolution of complex, nonlinear relationships among multiple parameters is critical to climate model improvement and validation. Making sense of the complexity and enormous volume of aerosol data and the diversity of types demands summarization as a practical way to facilitate widescale interpretation and extraction of patterns contained within the data. Efficient interpretation will require modern statistical and data mining techniques. Recent advances in these fields provide templates from which specific methods customized for aerosol science can be derived. Close collaboration between physical scientists, statisticians, and computer scientists is essential.

Systematic approaches to model evaluation. Approaches need to be established to compare chemical transport and radiative model outputs with observations in a systematic way, and rigorous metrics need to be generated for quantifying model and measurement discrepancies or deficiencies. The lack of integration between models and measurements is one of the main difficulties confronting the aerosol research community. Resolving these discrepancies requires establishing a comparison strategy that can isolate how well each process involved in the life cycle of aerosol layers is modeled. A sorting of air masses by source type potentially provides a way of establishing the spatial and temporal boundaries of the statistical ensembles used for data and model intercomparisons. Capturing the relationships between variables is necessary, and because many variables do not conform to Gaussian distributions, simple means and low-order moments are likely to be inadequate. The machinery of statistical hypothesis testing provides a useful guide to quantifying agreement between observed and modeled data distributions.

Establishing an accurate, consistent, and cohesive longterm record. A long-term system of aerosol observation requires sustainable accuracy, consistency, and resources. Continuity needs to be assured through robust calibration, validation, and measurement and model intercomparison programs. Cohesiveness of the complement of measurements is required, such that surface-based, airborne in situ, and satellite sensors observe the same parcels of air on the relevant temporal and spatial scales. Specific recommendations are presented in the following paragraphs, with further details provided in Diner et al. (2004).

Calibration AND VALIDATION. Research and technologies need to be invested in to improve sensor calibration and to develop systematic methods for validating aerosol optical and microphysical parameters obtained from remote sensing instruments. An important component of PARAGON is the development and application of techniques for the consistent evaluation of the uncertainties in measured and modeled aerosol properties. Independent observations and analyses for each key climate parameter are essential due to the difficulty of achieving climate quality accuracy with most instruments, the need to verify surprising climate change results, and the large economic and social impacts of climate change. Improvements in calibration accuracy and stability are required broadly across climate instruments. Closure experiments, which seek agreement between measured and calculated aerosol properties, and environmental snapshots from intensive field campaigns, are essential for validating results from instruments with wider areal coverage, and strategies for generating validation data on an ongoing basis need to be developed.

\section{MAINTENANCE AND AUGMENTATION OF SURFACE NETWORKS} AND IN SITU MEASUREMENTS ALOFT. Stable funding needs to be provided for surface-based radiometer, chemical, and lidar networks; coverage needs to be expanded into key undersampled areas; measurement capabilities need to be upgraded; and routine in situ aircraft observations should be established at selected sites, coincident with satellite overpasses. Accurate, qualityassured point observations of aerosol optical depth are widely available; however, uncertainties of columnintegrated microphysical parameters need to be routinely assessed against in situ observations. Increasing the density of surface stations should be considered in regions of high aerosol burden, high variability of aerosol properties, and over major source regions. Because it is important to sample midocean areas downwind of continental plumes, as well as in pristine areas that can provide useful validation of satellite retrievals, continued seaborne measurements of optical properties are needed. A lidar network with a proper global distribution of stations requires an extension of existing networks into data-sparse regions (e.g., the Southern Hemisphere), a transition to more 
homogeneous and more advanced instruments, and the installation of a common data-quality assurance program. Pure backscatter lidars will improve coverage in regions where it is difficult to operate more complex systems. Synergistic networks that combine optical depth, spectral directional sky radiance, microphysical property retrievals, and in situ capabilities need to be expanded. Several enhancements in chemical sampling data are essential to evaluating and improving CTMs. A program of sustained airborne measurements will be particularly helpful in supporting process studies, validation, and demonstrations of coherency between model predictions and remote sensing observations.

ReMOTE SENSING TECHNOLOGY ADVANCEMENTS. Advanced satellite imagers and lidars need to be developed to reduce indeterminacies in aerosol microphysical property retrievals, and the sensitivity of passive methods to height information where active measurements are unavailable needs to be investigated. Combining multispectral, multiangular, and polarimetric imaging techniques into a unified sensor can minimize uncertainties in column-integrated aerosol properties, owing to each method's unique sensitivities to particle microphysics. Lidars can provide the most accurate measurements of the vertical distribution of aerosol absorption, but the natural variability of tropospheric aerosol makes a pure backscatter lidar approach problematic due to ambiguities in separating backscatter and extinction. High spectral resolution and Raman lidar techniques derive profiles of these two quantities much more directly. The former approach is on a credible technology path toward space-based deployment, and the latter is widely viewed as the best method for the implementation of a ground-based network. Although passive methods of constraining aerosol height (e.g., imaging stereoscopy, multiangle near-UV observations, and oxygen A-band methods) are unlikely to provide the kind of detailed profiles achievable with lidars, they can provide wide spatial coverage much more readily, thus filling in gaps needed to constrain CTMs.

SATELLITE MISSION DESIGN. A systems approach needs to be adopted for the development of new satellite missions, factoring the integration of satellite measurements, surface and suborbital data, and models explicitly into instrument and mission design. Orbit selection affects satellite instrument capabilities, such as the sampling frequency of given locations (e.g., surface and in situ instrument emplacements), global coverage time, and synergy between passive and ac- tive approaches. Because even highly capable imagers cannot provide all the necessary measurements (e.g., details of aerosol chemistry), instrument and mission designs should take into account how they will be used in conjunction with other observational approaches, such as in situ samplers. In addition, because surfacebased lidars offer good temporal coverage, trade-offs in the placement and number of surface sites vis-àvis space-based lidars need to be examined, taking into account what is realistically achievable from space. This systems view requires taking into account how the various elements of a global observing network are to be designed and situated, and demands a level of coordination and planning that currently does not exist.

CONCLUSIONS. The PARAGON concept is designed to dramatically improve our understanding of the climate system's sensitivity to aerosols by revolutionizing the integration and coordination of aerosol research. Two principal points are highlighted, namely, that it is essential to 1) establish the means to evaluate and combine disparate data sources for maximum scientific benefit, and 2) establish a sustained, cohesive, and validated system to facilitate the transformation of information into knowledge. Four technical and practical challenges, and nine recommendations to address them, have been identified and are described in greater detail in the accompanying papers. A mapping between the challenges and recommendations is summarized in Table 1 . Our conclusions are intended to provide guidance for agencies that sponsor research, instrument development and deployment, and modeling. They are also intended to stimulate focus upon specific issues requiring detailed follow-up in order to implement the PARAGON recommendations (see the sidebar, "Questions to stimulate further study"). Pathways to resolving many of these questions are suggested in this series of papers.

Achieving the PARAGON vision requires the establishment of multidisciplinary, interagency, and international partnerships in order to advance our fundamental scientific understanding of aerosol patterns and processes. The concept involves a marriage of aerosol observations with assimilation and chemical transport modeling, information technology, geospatial statistics, and data mining research. An initiative of this breadth is necessary for establishing the quantitative role of aerosols in the earth system. If this role is as significant as some studies suggest, then this initiative will also be required to produce the technical basis for assessing the impact of regulatory choices on aerosol formation and concentrations. Without it, the 


\begin{tabular}{|c|c|c|c|c|}
\hline \multirow[b]{2}{*}{$\begin{array}{l}\text { Subject of } \\
\text { recommendation }\end{array}$} & \multicolumn{4}{|c|}{ Challenge area } \\
\hline & Data diversity & $\begin{array}{l}\text { Data quality } \\
\text { limitations }\end{array}$ & $\begin{array}{c}\text { Aerosol mesoscale } \\
\text { variability }\end{array}$ & $\begin{array}{l}\text { Cloud ubiquity } \\
\text { and heterogeneity }\end{array}$ \\
\hline $\begin{array}{l}\text { Data interoperability/ } \\
\text { computational infrastructure }\end{array}$ & $\sqrt{ }$ & $\sqrt{ }$ & $\sqrt{ }$ & \\
\hline Data integration & $\sqrt{ }$ & $\sqrt{ }$ & $\sqrt{ }$ & $\sqrt{ }$ \\
\hline Algorithm development & $\sqrt{ }$ & & & \\
\hline Summarization/mining & $\sqrt{ }$ & & & $\sqrt{ }$ \\
\hline Model evaluation & $\sqrt{ }$ & $\sqrt{ }$ & $\sqrt{ }$ & \\
\hline Calibration/validation & & $\sqrt{ }$ & $\sqrt{ }$ & \\
\hline Surface and in situ measurements & $\sqrt{ }$ & $\sqrt{ }$ & $\sqrt{ }$ & $\sqrt{ }$ \\
\hline Remote sensing technology & $\sqrt{ }$ & $\sqrt{ }$ & $\sqrt{ }$ & $\sqrt{ }$ \\
\hline Spacecraft mission design & & & $\sqrt{ }$ & $\sqrt{ }$ \\
\hline
\end{tabular}

scientific and policy communities will continue to struggle with understanding the full impact of complex aerosol processes on global and regional climate change and air quality.

ACKNOWLEDGMENTS. Support from the National Aeronautics and Space Administration, the National Oceanic and Atmospheric Administration, the National Science Foundation, and the U.S. Department of Energy is gratefully acknowledged. The research of D. J. Diner, A. J. Braverman, R. Davies, R. A. Kahn, J. V. Martonchik, and R. T. Menzies was conducted at the Jet Propulsion Laboratory, California Institute of Technology, under contract with NASA. The research of T. P. Ackerman was conducted at the Pacific Northwest National Laboratory under contract with JPL. T. L. Anderson and R. J. Charlson acknowledge support from the National Science Foundation (Grant ATM-0138250). The research work of O. Torres was funded by NASA, and conducted at the Joint Center for Earth Systems Technology, University of Maryland, Baltimore County.

\section{APPENDIX: DEFINITIONS OF FREQUENTLY USED TERMS.}

Active remote sensing: A technique for probing a target from a distance by illuminating it with a generated electromagnetic signal (such as a laser pulse or microwave beam), and measuring the returned signal.

Aerosols: Airborne solid or liquid particles, typically in the size range of $0.001-10 \mu \mathrm{m}$ in radius. Anthropogenic aerosols are those arising from human activities.
Aerosol direct radiative forcing: The portion of the global radiation budget attributable to scattering and absorption by both natural and anthropogenic aerosol particles.

Aerosol indirect radiative forcing: The portion of the global radiation budget attributable to the effects of aerosols on the radiative properties of clouds, on cloud lifetimes, and on cloud amounts.

Aerosol optical depth: A measure of aerosol amount, formally the light extinction produced by aerosol scattering and absorption along a vertical path, numerically equal to the negative logarithm of the directbeam transmittance vertically through an atmospheric column.

Data assimilation: The process of controlling the evolution of a modeled system by incorporating observational data to continuously update the initial conditions of the model equations.

Chemical transport model: A computer model describing the transport and transformation of atmospheric gases and aerosol particles based upon prescribed meteorological fields, source inventories, and representations of chemical and physical processes.

Cloud condensation nuclei: Aerosol particles that act as seeds for the formation of clouds through the condensation of water molecules onto their surfaces.

Data interoperability: The ability to process and exchange data from multiple platforms collected at disparate spatial and temporal scales. 
Data interoperability and computational infrastructure: How can advances in information technologies be exploited to create an aerosol "virtual observatory?" What standards need to be established? Can enhanced computational power improve the spatial and temporal resolution of global models?

Data integration: What methodologies are needed to routinely merge in situ aircraft data with spaceborne and surface-based radiometers and lidars? How can geospatial statistics, assimilation, and transport model approaches be used synergistically? Is it feasible to use such approaches to merge data acquired with different spatial and temporal sampling? How should conflicting data be dealt with? What are the most effective methods of incorporating subgrid-scale information into global models?

Multisensor algorithm development: What algorithmic improvements can significantly enhance the scientific return from existing sensors by combining their data together into joint retrievals? What are the limits of current observations in constraining global aerosol optical depth and its anthropogenic fraction, and can combinations of approaches reduce uncertainties? What observational gaps remain even when current capabilities are combined?

Data summarization and mining: What are the most effective strategies for mining multivariate datasets, such as joint aerosol and cloud distributions and microphysical properties? Are there sufficient correlations within airmass types to enable the use of classification methods to map them? How can new data mining methods, such as modern approaches to supervised and unsupervised learning, be used to identify and characterize patterns in the data?

Systematic approaches to model evaluation: How do we develop a model-testing environment that goes beyond simple comparisons of monthly mean properties from model/data and evaluates joint distributions of variables within large ensembles sorted by aerosol source region and/or aerosol type? Can data mining be used to establish the spatial and temporal boundaries of such ensembles? What criteria should be used to judge whether observed and modeled data agree or disagree?

Calibration and validation: How can calibration accuracies of spaceborne remote sensing imagers be improved? What standards need to be established so that instrument and model differences can be resolved, and continuity achieved, as sensor technology and computational capabilities evolve? What standards can be established to enable quantitative evaluation of high-level products derived from remote sensing?

Maintenance and augmentation of surface networks and in situ measurements aloft: What essential aerosol measurements are missing from current observations, and what priority should be assigned to acquiring them? What steps need to be taken to maintain and upgrade existing networks? What are the trade-offs in number and placement of an expanded complement of instruments? Where are the optimal locations to routinely deploy aircraft for atmospheric in situ vertical profiling? Where are the most critical undersampled locations? Can a routine network of mobile, oceanic radiometers be established? What other mobile platforms might be considered over land and ocean?

Remote sensing technology advancements: What are the technical and cost trade-offs between an expanded surface lidar network and lidars in space? Can techniques such as stereoscopy, multiangle UV sounding, oxygen A-band spectroscopy, or other passive techniques for determining aerosol height information be of quantitative value in the absence of satellite or surface lidar data? How can these approaches best complement one another? What technology demonstration experiments (e.g., from satellite, aircraft, and ground-based sensors, or by developing new aircraft sensors) need to be performed to achieve verifiable accuracy gains in the retrieval of optical depth and single scattering albedo? How do we judge the value of, for example, doubling the accuracy in optical depth versus doubling the accuracy of particle size or single scattering albedo?

Satellite mission design: What are the performance and cost trade-offs in choices of orbit altitudes, equator-crossing times, and numbers of platforms; and how will their results augment and complement suborbital and surface strategies? What rigorous, quantitative metrics can be established in order to provide instrument designers with spatial coverage and revisit frequency requirements? How do cloud and aerosol missions need to complement one another, and surface-based and airborne measurements, to deal with indirect forcing? 
Extensive aerosol properties: Quantities that are functions of particle amount, such as aerosol concentration or optical depth.

Extinction: The removal of electromagnetic radiation from a propagating beam, through both absorption and scattering.

General circulation model: A computer model describing the evolution of climate variables, such as temperature and precipitation, driven by physical equations describing the exchange of energy and water between the ocean, land surface, and atmosphere. These models usually aim to conserve energy, momentum, and mass on a rotating sphere.

Geospatial statistics: A discipline dealing with the statistical behavior of geographically referenced data.

Grid computing: An architecture where computations are performed on geographically distributed machines, and data are transferred over high-speed networks.

Intensive aerosol properties: Quantities that are independent of aerosol amount, such as particle shape or refractive index.

Mesoscale: A spatial scale from tens to hundreds of kilometers.

Middleware: Software services that mediate data access and exchange between archives and users.

Passive remote sensing: A technique for probing a target from a distance by observing reflected sunlight or radiation emitted by the target in any part of the electromagnetic spectrum.

Refractive index: A complex (real and imaginary) parameter describing the propagation of light in a medium, for example, an aerosol particle. Its real part is the ratio of the speed of light in a vacuum to the speed within the medium; its imaginary part is related to the absorptivity of the medium.

Scattering phase function: The probability per unit solid angle that a particle scatters a photon into a particular direction relative to the direction of the incident beam.

Single scattering albedo: Given an interaction between a photon and an aerosol particle, the probability that the photon is scattered into some direction, rather than absorbed.

Supersaturation: An atmospheric condition where the relative humidity exceeds $100 \%$.

Validation: The process of establishing the accuracy of a retrieved quantity through a comparison with independent measurements having known uncertainties.

\section{REFERENCES}

Ackerman, T. P., and Coauthors, 2004: Integrating and interpreting aerosol observations and models within the PARAGON framework. Bull. Amer. Meteor. Soc., 85, 1523-1533.

Baltensperger, U., and Coauthors, 2003: WMO/GAW aerosol measurement procedures: Guidelines and recommendations. WMO Tech. Doc. 1178, 72 pp.

Charlson, R. J., 2001: Extending atmospheric aerosol measurements to the global scale. IGAC Newsl., 25, International Global Atmospheric Chemistry Project, 11-14.

Demerjian, K. L., 2000: A review of national monitoring networks in North America. Atmos. Environ., 34, 1861-1884.

Diner, D. J., and Coauthors, 2004: Using the PARAGON framework to establish an accurate, consistent, and cohesive long-term aerosol record. Bull. Amer. Meteor. Soc., 85, 1535-1548.

DOE, 2001: Tropospheric Aerosol Program-Program plan. U.S. Department of Energy Rep. DOE/SC-0034, $98 \mathrm{pp}$.

Duce, R. A., and Coauthors, 1993: Protecting Visibility in National Parks and Wilderness Areas. National Academy Press, 316 pp.

Heintzenberg, J., H.-F. Graf, R. J. Charlson, and P. Warneck, 1996: Climate forcing and the physicochemical life cycle of the atmospheric aerosol-Why do we need an integrated, interdisciplinary global research programme? Beitr. Phys. Atmos., 69, 261271.

Kahn, R. A., and Coauthors, 2004: Aerosol data sources and their roles within PARAGON. Bull. Amer. $\mathrm{Me}$ teor. Soc., 85, 1511-1522.

Kaufman, Y. J., and T. Nakajima, 1993: Effect of Amazon smoke on cloud microphysics and albedoAnalysis from satellite imagery. J. Appl. Meteor., 32, 729-744.

Mahoney, J. R., and Coauthors, 2003: The U.S. Climate Change Program: Vision for the program and highlights of the scientific strategic plan. Climate Change Science Program and the Subcommittee on Global Change Research Rep., 41 pp. [Available online at www.climatescience.gov/Library/stratplan2003/vision/ccsp-vision.pdf.]

Molina, M. M., and Coauthors, 2001: Global Air Quality: An Imperative for Long-Term Observational Strategies. National Research Council, 51 pp.

Penner, J. E., and Coauthors, 1994: Quantifying and minimizing uncertainty of climate forcing by anthropogenic aerosols. Bull. Amer. Meteor. Soc., 75, 375400 . 
—- and Coauthors, 2001: Aerosols, their direct and indirect effects. Climate Change 2001: The Scientific Basis, J. T. Houghton et al., Eds., Cambridge University Press, chapter 5 .

Ramanathan, V., P. J. Crutzen, J. T. Kiehl, and D. Rosenfeld, 2001: Aerosols, climate, and the hydrological cycle. Science, 294, 2119-2124.

— , and Coauthors, 2002: The National AerosolClimate Interactions Program (NACIP): A national research imperative. NACIP Scientific Steering Committee White Paper, 11 pp. [Available online at wwwnacip.ucsd.edu/NACIPWhitePaperMay2102.pdf.]

Ramaswamy, V., and Coauthors, 2001: Radiative forcing of climate change. Climate Change 2001: The Scientific Basis, J. T. Houghton et al., Eds., Cambridge University Press, chapter 6.

Rosenfeld, D., 2000: Suppression of rain and snow by urban and industrial air pollution. Science, 287, 1793-1796.

Seinfeld, J. H., and Coauthors, 1996: A Plan for a Research Program on Aerosol Radiative Forcing and Climate Change. National Research Council, 161 pp.

— and Coauthors, 2004: Scientific objectives, measurement needs, and challenges motivating the PARAGON aerosol initiative. Bull. Amer. Meteor. Soc., 85, 1503-1509.

Trenberth, K. E., T. R. Karl, and T. W. Spence, 2002: The need for a systems approach to climate observations. Bull. Amer. Meteor. Soc., 83, 1593-1602.

Twomey, S. A., 1977: The influence of pollution on the shortwave albedo of clouds. J. Atmos. Sci., 34, 11491152. 